\title{
Prevalência de pré-obesidade, obesidade e práticas saudáveis e não saudáveis associadas
}

\author{
Prevalence of pre-obesity, obesity and associated healthy and unhealthy practices \\ Prevalencia de pre-obesidad, obesidad y prácticas saludables y no saludables asociadas \\ Vanessa da Silva do Nascimento ${ }^{1 *}$, Maria Devany Pereira ${ }^{1}$, Ivonete Moura Campelo ${ }^{1}$, Layonne de \\ Sousa Carvalho ${ }^{1}$, Cecília Maria Resende Gonçalves de Carvalho ${ }^{1}$, Marize Melo dos Santos ${ }^{1}$.
}

\begin{abstract}
RESUMO
Objetivo: Investigar em escolares a prevalência de pré-obesidade, obesidade e práticas saudáveis e não saudáveis associadas. Métodos: Foi aplicado questionário à cada participante contendo variáveis sociodemográficas, comportamentos saudáveis e não saudáveis e antropometria. Utilizou-se estatística descritiva a partir do "n" e proporções, além de análises de associações a partir do teste qui-quadrado, adotando significância $p<0,05$. O estudo foi aprovado pelo CEP-UFPI. Resultados: Participaram do estudo 255 escolares, com faixa etária de 10 a 17 anos, matriculados em escola urbana e rural do munícipio. A prevalência de excesso de peso foi de $20,0 \%$. A maioria dos adolescentes afirmou realizar refeições em frente as telas, assim como boa parte afirmou não substituir as principais refeições por lanches. A maioria referiu 0 consumo de refrigerante, independente do estado nutricional. Os adolescentes mais ativos apresentam estado nutricional adequado, sendo que a maioria dos adolescentes afirmou praticar atividade física. Conclusão: Embora a maioria tenha apresentado estado nutricional adequado, a prevalência de excesso de peso aproximou-se do encontrado na literatura. Destaca-se que estudantes da zona rural apresentaram maior prevalência de excesso de peso.
\end{abstract}

Palavras-chaves: Excesso de peso, Consumo alimentar, Atividade física.

\begin{abstract}
Objective: To investigate in schoolchildren the prevalence of pre-obesity, obesity and associated healthy and unhealthy practices. Methods: A questionnaire was applied to each participant containing sociodemographic variables, healthy and unhealthy behaviors and anthropometry. Descriptive statistics were used from the " $\mathrm{n}$ " and proportions, as well as association analyzes from the chi-square test, adopting significance $p<0.05$. The study was approved by CEP-UFPI. Results: The study included 255 students, aged 10 to 17 years, enrolled in urban and rural schools in the municipality. The prevalence of overweight was $20.0 \%$. Most teens said they had meals in front of the screens, just as most said they did not replace main meals with snacks. Most reported soda consumption, regardless of nutritional status. The most active adolescents have adequate nutritional status, and most adolescents reported physical activity. Conclusion: Although most had adequate nutritional status, the prevalence of overweight was close to that found in the literature. It is noteworthy that rural students had a higher prevalence of overweight.
\end{abstract}

Key words: Overweight, Food consumption, Physical activity.

1Universidade Federal do Piauí (UFPI), Teresina-PI. *E-mail: vanessanascimentox10@gmail.com

Financiado pelo Conselho Nacional de Desenvolvimento Científico e Tecnológico (CNPq), na Chamada CNPq/MCTIC № 016/2016.

SUBMETIDO EM: 9/2019

ACEITO EM: 10/2019

PUBLICADO EM: 12/2019 


\section{RESUMEN}

Objetivo: Investigar en escolares la prevalencia de pre-obesidad, obesidad y prácticas saludables y no saludables asociadas. Métodos: se aplicó un cuestionario a cada participante que contenía variables sociodemográficas, comportamientos saludables y no saludables y antropometría. Se utilizaron estadísticas descriptivas de la "n" y las proporciones, así como análisis de asociación de la prueba de ji al cuadrado, adoptando significancia $p<0.05$. El estudio fue aprobado por CEP-UFPI. Resultados: El estudio incluyó a 255 estudiantes, de entre 10 y 17 años, matriculados en escuelas urbanas y rurales del municipio. La prevalencia de sobrepeso fue del $20,0 \%$. La mayoría de los adolescentes dijeron que tenían comidas frente a las pantallas, al igual que la mayoría dijeron que no reemplazaban las comidas principales con bocadillos. La mayoría informó el consumo de refrescos, independientemente del estado nutricional. Los adolescentes más activos tienen un estado nutricional adecuado, y la mayoría de los adolescentes informaron actividad física. Conclusión: Aunque la mayoría tenía un estado nutricional adecuado, la prevalencia de sobrepeso era cercana a la encontrada en la literatura. Es de destacar que los estudiantes rurales tenían una mayor prevalencia de sobrepeso.

Palabras clave: Sobrepeso, Consumo de alimentos, Actividad física.

\section{INTRODUÇÃO}

As grandes mudanças no perfil nutricional de adolescentes brasileiros caracterizaram-se pela redução contínua da desnutrição entre 1975 a 2009 e aumento da prevalência de excesso de peso. De acordo com a Pesquisa de Orçamento Familiar (POF), realizada pelo Instituto Brasileiro de Geografia e Estatística (IBGE), o excesso de peso atingiu mais de $30 \%$ das crianças entre cinco e nove anos de idade e cerca de $20 \%$ da população entre 10 e 19 anos de idade (BRASIL, 2010).

Segundo a World Health Organization (WHO), pré-obesidade e obesidade são definidas como acúmulo excessivo de gordura com potencial prejuízo a saúde. No ano de 2000 , as estatísticas já apontavam que a obesidade se tornaria um problema para a saúde pública em escala mundial (WHO, 2000).

Isso foi visto mais tarde, em 2016, quando WHO mostrou que a prevalência mundial de obesidade quase triplicou entre 1975 e 2016. Seguindo tendências globais, a pré-obesidade e a obesidade constituem um distúrbio nutricional crescente no continente latino, notado, especialmente, entre a população abaixo de 20 anos de idade, onde o excesso de peso assumiu proporções epidêmicas (WHO, 2018).

Dentre as suas causas, tem-se que a pré-obesidade e a obesidade são condições de etiologia multifatorial e que estas sofrem influências de fatores biológicos, psicológicos, socioeconômicos e sócio-comportamentais (KUMAR S e KELLY AS, 2017; WANG S, et al. 2017).

Nesse sentido, o aumento alarmante de adolescentes com excesso de peso está ligado às mudanças de hábitos da população, voltada para uma dieta inadequada, com mais disponibilidade e ingestão de alimentos com alto teor energético, mudança no estilo de vida ativa para sedentária e aumento das porções alimentares (SAHOO K, et al. 2015).

A detecção de obesidade nessa população infanto-juvenil era incomum décadas atrás nos países em desenvolvimento e em situação socioeconômica precária (RECH RR e HALPERN R, 2011). Entretanto, em decorrência do elevado crescimento dessa prevalência no mundo, este fenômeno tem-se tornado preocupante. Esse excesso de peso, além de ser fator de risco para uma série de agravos à saúde física, também pode acarretar prejuízos, a longo prazo, como obesidade na vida adulta e complicações que acompanham esse quadro (GURNANI M, et al. 2015).

Além disso, outras consequências da obesidade nessa fase de vida podem ser notadas, como aumento da diabetes melittus tipo 2, esteatose hepática e problemas ortopédicos, bem como maior risco para complicações cardiovasculares como hipertensão, dislipidemia, aterosclerose e doença coronariana que resulta em redução da expectativa de vida (THE NS, et al. 2010; WHO, 2018). 
Tendo em vista que o aumento do excesso de peso em crianças e adolescentes tem incitado à busca de possíveis fatores associados e à forma eficaz de combate e prevenção do problema, estudos têm sido cada vez mais realizados. Em 2016, o Estudo de Riscos Cardiovasculares em Adolescentes (ERICA) realizado por Bloch KV, et al. (2016), foi o primeiro estudo brasileiro com representatividade nacional para estimar o estado nutricional de adolescentes, neste observou-se que 25,0\% dos adolescentes brasileiros estão com excesso de peso. Entretanto, poucos estudos têm investigado municípios de pequeno porte. Nesse sentido, o objetivo do presente estudo foi investigar a prevalência de pré-obesidade e obesidade e sua associação com práticas saudáveis e não saudáveis de um município de pequeno porte, no estado do Piauí.

\section{MÉTODOS}

O presente estudo advém da pesquisa intitulada "Estudo de práticas de vida saudável com estudantes de ensino fundamental e médio na perspectiva da promoção da soberania e segurança alimentar e nutricional", financiado pelo Conselho Nacional de Desenvolvimento Científico e Tecnológico (CNPq), na Chamada CNPq/MCTIC № 016/2016. Trata-se de um estudo descritivo, tipo transversal. Realizado durante os meses de agosto a novembro de 2018.

Para a inclusão das escolas e turmas foi feito sorteio aleatório, envolvendo escolas públicas da zona urbana e rural. O sorteio baseou-se em 10\% do total de escolas do município (62), resultando em 6 escolas (4 urbanas e 2 rurais). O cálculo amostral resultou em um número mínimo de 170 escolares para inclusão no estudo.

Para participar do estudo os adolescentes atenderam aos seguintes critérios de elegibilidade: adolescentes entre dez a dezessete anos de idade; adolescentes que aceitassem participar da pesquisa e assinassem o Termo de Assentimento (TA); pais que aceitassem a participação dos seus filhos na pesquisa e assinassem o Termo Consentimento Livre e Esclarecido (TCLE).

Foram investigadas as variáveis sociodemográficas (sexo, idade, tipo de escola), práticas saudáveis e não saudáveis (deslocamento para a escola, realização de atividade física, realização de refeições em frente a telas de televisão e/ou computador e outros eletrônicos, consumo de lanches rápidos nas principais refeições, consumo de refrigerantes) e antropométricas (baixo peso, eutrófia, pré-obesidade e obesidade). Todos os dados foram coletados em visitas às escolas, com uso de celular e software aplicativo (zoho forms). Para as variáveis sociodemográficas e práticas saudáveis e não saudáveis, a coleta dos dados foi por meio da aplicação de um questionário. Os estudantes foram submetidos à avaliação antropométrica, realizada por avaliadores previamente treinados.

Utilizou-se os parâmetros, peso e estatura, para obtenção do índice de massa corporal para idade (IMC/idade). Para a análise, o IMC foi convertido em percentis por idade e sexo e classificados de acordo com os critérios propostos pela WHO em 2007 e adotados pelo Ministério da Saúde (BRASIL, 2011).

O projeto foi submetido e aprovado pelo Comitê de Ética em Pesquisa (CEP) da Universidade Federal do Piauí (UFPI), seguindo as diretrizes regulamentadoras de pesquisas envolvendo seres humanos (Resolução MS/CNS no 466/2012), sob parecer consubstanciado no 2.379.672.

Para análise dos dados foi utilizado o programa estatístico R, versão 2.11.1. Para a determinação de prevalência, utilizou-se a estatística descritiva, a partir do "n" e proporções. As associações entre as variáveis de estudo foram verificadas a partir do teste qui-quadrado com tabelas de continência, adotando-se nível de significância de $5 \%$.

\section{RESULTADOS}

Participaram do estudo 255 adolescentes, sendo 60,4\% ( $n=154)$ das escolas da zona urbana e 39,6\% $(n=101)$ das escolas da zona rural, destes, 116 são do sexo masculino $(45,5 \%)$ e 139 do sexo feminino (54,5\%), com faixa etária de 10 a 17 anos, no qual a maioria apresenta de 10 e 12 anos (47,9\%). Na Tabela 1 estão as características gerais da amostra investigada. 
Tabela 1 - Caracterização sociodemográfica segundo sexo, faixa etária e tipo de escola.

\begin{tabular}{lll}
\hline Caracterização sociodemográfica & N & $\%$ \\
\hline Sexo & 116 & \\
\hline Masculino & 139 & 54,5 \\
Feminino & & \\
\hline Idade & 122 & 47,9 \\
\hline $10-12$ & 111 & 43,5 \\
$13-14$ & 22 & 8,6 \\
$15-17$ & & 60,4 \\
\hline Tipo de escola & 154 & 39,6 \\
\hline Urbana & 101 & $\mathbf{1 0 0 , 0}$ \\
\hline Rural & $\mathbf{2 5 5}$ & \\
\hline Total & &
\end{tabular}

Fonte: Nascimento VS, et al., 2019.

A prevalência de excesso de peso encontrado foi de $20,0 \%$, estando $12,9 \%$ e $7,1 \%$ dos escolares nas faixas de pré-obesidade e obesidade, respectivamente. Alunos provenientes de escolas rurais apresentaram maior prevalência de excesso de peso (Tabela 2).

Tabela 2 - Classificação do IMC por idade segundo tipo de escola.

\begin{tabular}{lcccccc}
\hline & \multicolumn{2}{c}{ Zona urbana } & \multicolumn{2}{c}{ Zona rural } & \multicolumn{3}{c}{ Total } \\
Classificação IMC/I & no & $\%$ & no & $\%$ & no & $\%$ \\
\hline Baixo peso & 5 & 3,2 & 10 & 9,9 & 15 & 5,9 \\
Eutrofia & 121 & 78,6 & 68 & 67,3 & 189 & 74,1 \\
Pré-obesidade & 18 & 11,7 & 15 & 14,9 & 33 & 12,9 \\
Obesidade & 10 & 6,5 & 8 & 7,9 & 18 & 7,1 \\
\hline Total & $\mathbf{1 5 4}$ & $\mathbf{1 0 0 , 0}$ & $\mathbf{1 0 1}$ & $\mathbf{1 0 0 , 0}$ & $\mathbf{2 5 5}$ & $\mathbf{1 0 0 , 0}$
\end{tabular}

Legenda: IMC/l: Índice de Massa Corporal por Idade. Fonte: Nascimento VS, et al., 2019.

Para fins de associação entre o estado nutricional e as práticas saudáveis e não saudáveis, levou-se em consideração apenas eutrofia, pré-obesidade e obesidade, eliminando o baixo peso apara a idade, totalizando um "n" de 240 estudantes.

Tabela 3 - Associação entre variáveis relativas a práticas não saudáveis e estado nutricional dos alunos.

\begin{tabular}{|c|c|c|c|c|c|}
\hline \multirow{3}{*}{ Variáveis de consumo } & \multicolumn{4}{|c|}{ Estado Nutricional $(\mathrm{n}=\mathbf{2 4 0})$} & \multirow{3}{*}{ Valor de $p$} \\
\hline & \multicolumn{2}{|c|}{ Eutrofia } & \multirow{2}{*}{\multicolumn{2}{|c|}{ Excesso de Peso }} & \\
\hline & $n^{0}$ & $\%$ & & & \\
\hline \multicolumn{6}{|l|}{ Refeições em frente as telas } \\
\hline Às vezes & 33 & 13,8 & 8 & 3,3 & \multirow{3}{*}{0,9493} \\
\hline Não & 45 & 18,8 & 12 & 5,0 & \\
\hline Sim & 111 & 46,2 & 31 & 12,9 & \\
\hline \multicolumn{6}{|l|}{ Lanches rápidos nas refeições } \\
\hline Às vezes & 26 & 10,8 & 8 & 3,3 & \multirow{3}{*}{0,7884} \\
\hline Não & 98 & 40,8 & 28 & 11,7 & \\
\hline Sim & 65 & 27,1 & 15 & 6,2 & \\
\hline \multicolumn{6}{|l|}{ Refrigerantes } \\
\hline 1 ou $2 \times \mathrm{p} / \mathrm{sem}$ & 80 & 33,3 & 22 & 9,2 & \multirow{4}{*}{0,464} \\
\hline 2 ou $3 \times \mathrm{p} / \mathrm{sem}$ & 28 & 11,7 & 8 & 3,3 & \\
\hline Diariamente & 21 & 8,8 & 2 & 0,8 & \\
\hline Nunca/raramente & 60 & 25,0 & 19 & 7,9 & \\
\hline
\end{tabular}

Fonte: Nascimento VS, Pereira MD, Campelo IM, et al., 2019. 
Tabela 4 - Associação entre variáveis relativas às práticas saudáveis e estado nutricional dos alunos.

\begin{tabular}{|c|c|c|c|c|c|}
\hline \multirow{3}{*}{ Variáveis } & \multicolumn{4}{|c|}{ Estado Nutricional $(n=240)$} & \multirow{3}{*}{ Valor de $p$} \\
\hline & \multicolumn{2}{|c|}{ Eutrofia } & \multicolumn{2}{|c|}{ Excesso de Peso } & \\
\hline & no & $\%$ & no & $\%$ & \\
\hline \multicolumn{6}{|c|}{ Deslocamento para a escola } \\
\hline Andando/caminhando & 56 & 23,3 & 17 & 7,1 & \multirow{3}{*}{0,8176} \\
\hline Bicicleta & 50 & 20,8 & 14 & 5,8 & \\
\hline Carro/ônibus/moto & 83 & 34,6 & 20 & 8,3 & \\
\hline \multicolumn{6}{|c|}{ Realização de atividade física } \\
\hline $\operatorname{Sim}$ & 125 & 52,1 & 36 & 15,0 & 0,6655 \\
\hline Não & 64 & 26,7 & 15 & 6,2 & \\
\hline
\end{tabular}

Fonte: Nascimento VS, et al., 2019.

Embora o maior percentual de excesso de peso tenha sido para a resposta "sim" quanto a refeições em frente as telas $(12,9 \%)$, resposta "não" para lanches rápidos nas principais refeições $(11,7 \%)$ e resposta "1 ou $2 \times \mathrm{p} /$ sem" para consumo de refrigerantes $(9,2 \%)$, não houve diferenças entre as variáveis relativas às práticas alimentares não saudáveis e estado nutricional $(p>0,05)$ (Tabela 3).

No que diz respeito as variáveis de atividades, o maior percentual de excesso de peso foi para aqueles que se deslocam de forma ativa (bicicleta e andando) (12,9\%). A prática de atividade física apresentou percentual mais prevalente de excesso de peso para a resposta "sim" (15\%) (Tabela 4).

\section{DISCUSSÃO}

Identificar a associação de práticas saudáveis e não saudáveis com prevalência de pré-obesidade e obesidade em escolares, permite intervenção precoce além de minimizar risco de desenvolvimento de doenças crônicas no futuro (GURNANI M, et al. 2015).

O presente estudo observou excesso de peso em escolares da zona urbana e rural, sendo a última de maior percentual, resultados semelhantes foram observados por Panazzolo PR, et al. (2014). Estes dados sugerem que a maior propensão ao excesso de peso pode variar entre diferentes populações e é para aqueles que residem longe dos centros urbanos. Visto que, quando comparadas ao estudo de Bloch KV, et al. (2016), com escolares de 12 a 17 anos, em várias macrorregiões do Brasil, Teresina estava entre as prevalências mais baixas de sobrepeso e obesidade $(6,0 \%)$.

Em relação a realização de refeições em frente as telas, esses dados são coincidentes aos encontrados por Oliveira JS, et al. (2016) e Ramos MLM, et al. (2013), onde observaram resultados semelhantes ao do presente estudo, demonstrando que esse costume vem se tornando cada vez mais frequente entre os adolescentes, independentemente do estado nutricional. Uma hipótese para isso pode estar relacionada ao nível de instrução e a rotina dos pais, tornando mais difícil a conscientização dos adolescentes quanto a realização de refeições à mesa. Não se observou diferenças significativas entre excesso de peso e o consumo de refeições em frente as telas, embora seja sabido que a relação dos adolescentes com as telas é evidenciada na literatura, conforme relado por Musaiger AO e Kalam F (2014).

Resultados similares foram apresentados por Oliveira JS, et al. (2016) no ERICA, quando analisado essa prática em todas as regiões do País, mais da metade dos adolescentes referiu consumir refeições em frente a televisão, assim como a maior parte dos adolescentes referiu consumir petiscos em frente a telas às vezes e aproximadamente $40,0 \%$ quase sempre ou sempre adotam tal prática.

Quando questionados sobre o ato de substituir as grandes refeições por lanches rápidos, evidenciou-se que a maioria dos escolares não praticam tal hábito.

Resultados similares foram encontrados na literatura, como o estudo realizado por Santos MM, et al. (2014), com adolescentes de 10 a 14 anos de idade. Já Ramos MLM, et al. (2013), trabalhando com adolescentes na mesma faixa etária, demonstraram prevalência maiores que as encontradas nesta investigação, para consumo de fast foods. Mesmo os escolares que praticavam o ato de substituir as 
refeições por lanches, serem uma parcela menor, ainda era um número considerável. Isto indica que há uma possível homogeneização no padrão alimentar e que as comunidades rurais estão adquirindo e ingerindo os mesmos alimentos consumidos nos centros urbanos, como verificado por Reinado EDF, et al. (2015).

Ainda sobre esse ponto, viu-se que o excesso de peso foi encontrado em maior percentual entre os alunos que relataram não fazer tal substituição, apresentando não associação. Apesar do resultado seja contrário ao que a literatura tem apresentado, estudos de D'avila GL, et al. (2016);

Neto ACB, et al. (2015), também observaram resultados semelhantes aos do presente estudo. Esses achados podem ser explicados pela frequência elevada de excesso de peso, interferindo no comportamento alimentar ou pelo subrelato da não substituição de refeições por lanches.

Quanto ao consumo de refrigerantes, pode-se notar que grande parcela dos escolares relatou consumi-lo com frequência. Uma hipótese para este achado é que o baixo nível socioeconômico pode interferir em melhores escolhas pelos escolares, mesmo em locais distantes dos grandes centros urbanos, o menor custo de alimentos não saudáveis contribui ainda mais para o quadro de excesso de peso, que vem se expandido pelo país, assim como identificou Musaiger AO, e Kalam F, (2014) em seu estudo.

Resultados similar aos encontrados também foi observado por Pintos RP, et al. (2016), no qual a associação entre as variáveis frequência do consumo de refrigerante e excesso de peso não apresentaram diferenças estatisticamente significativas, tornando necessário a realização de mais estudos a fim de explicar tal resultado.

Para Souza AM, et al. (2016) esse maior consumo de refrigerantes, outras bebidas açucaradas e fast-food e inatividade física, está diretamente relacionado ao excesso de peso em adolescentes, abordado no ERICA (2016), sua pesquisa mostra que essa prática interfere diretamente em comportamentos alimentares saudáveis, deixando de lado 0 ato de fazer as refeições com a família, além de substituir água por bebidas açucaradas. Com relação às práticas saudáveis, ao analisar o meio de deslocamento trajeto casa/escola com excesso de peso em adolescentes, não foi possível associação significativa, assim como é observado em pesquisa no estado Santa Catarina por Kneipp C, et al. (2015).

De acordo com Sousa S, et al. (2015), existem fatores negativos e fatores positivos que podem interferir no deslocamento ativo de casa para escola, o que sugere que aspectos como proximidade casa-escola, pratica de atividade física, longas distâncias, cansaço, insegurança, trânsito e violência influenciaram na percepção dos adolescentes em relação ao meio deslocamento escolhido. Tornando necessário, como mencionado por Souza S, et al. (2018), que o ambiente físico e infraestrutura que envolve o cotidiano do trajeto realizado pelos adolescentes à escola seja agradável, convidativo, seguro e favorável, a fim de potencializar a mobilidade ativa destes.

No que concerne a prática de atividade física, o estudo verificou que a maior parte da amostra estudada afirma fazê-la. Esses resultados foram semelhantes aos obtidos por Rangel SRV, et al. (2015) e Basto F, et al. (2015). Diferentemente, Alberto AAD e Junior AJF, (2015), Barbosa SC, et al. (2016) e Dumith SC, et al. (2016) verificaram que a prevalência de adolescentes fisicamente ativos na amostra ainda era baixa.

Observa-se que os estudos encontrados apresentam diferentes resultados, tal fenômeno pode ser justificado pelas diferenças no âmbito sociocultural e comportamental dos adolescentes, assim como apresentado por Cureau FV et al (2016), mostrou que mais da metade dos adolescentes brasileiros estão inativos fisicamente, além do fato de um a cada quatro adolescentes não praticarem atividade física no lazer. De forma que a prevalência de inatividade física foi maior na região Nordeste e menor na região Sul. Podese se inferir a diferença entre a capital e uma cidade do interior do estado.

Quanto a associação entre a prática de atividade física e excesso de peso, embora alguns estudos relatem associação entre essas variáveis, no trabalho de Alazzeh AY, et al. (2018), não houve associação significativa dos mesmos. Resultados semelhantes foram obtidos em outros estudos, como na cidade de Caracol/PI (MONTEIRO AR, et al. 2016), Ribeirão Preto/SP (PINTOS RP, et al. 2016) e Florianópolis/SC (D'AVILA GL, et al. 2016), onde também não foram observadas associações significativas quando analisadas essas 
variáveis. Achados como esses é difícil de ser explicado, pois não foi avaliado no presente estudo, o consumo alimentar dos adolescentes, o que pode estar interferindo nos resultados, uma vez que a maior prevalência tanto de excesso de peso como de eutrofia foi para aqueles que realizaram atividade física.

Compreender as relações entre obesidade e diferentes características socioeconômicas e comportamentais pode ajudar na elaboração de estratégias mais eficazes de prevenção da obesidade em jovens, de forma a reduzir suas complicações e garantir não só a tendência de crescimento da expectativa de vida, mas também a qualidade de vida das futuras gerações (BLOCH KV et al. 2016).

O estudo contribui com o que é apresentado na literatura com relação a fatores comportamentais e o aumento alarmante de excesso de peso em adolescentes. Vale destacar que estudos atuais nessa perspectiva com escolares do interior do estado, zona rural e urbana, ainda são escassos, portanto, a contribuição para futuros trabalhos e comparações com novas pesquisas é de suma importância.

\section{CONCLUSÃO}

Na prevalência de excesso de peso não foi observada associação com práticas saudáveis e não saudáveis. Contudo, essas práticas apresentaram maior prevalência para aqueles escolares com estado nutricional eutrófico. Embora a maioria tenha apresentado estado nutricional adequado, destaca-se que a prevalência de excesso de peso aproximou-se do encontrado no ERICA (25,0\%). Estudantes da zona rural apresentaram maior prevalência de excesso de peso.

\section{REFERÊNCIAS}

1. ALBERTO AAD, JUNIOR AJF. Prevalência de inatividade física e sua associação com variáveis sociodemográficas em adolescentes do Município de Macapá/AP. Revista Brasileira de Ciência e Movimento, 2015; 23(4) 80-93.

2. ALAZZEH AY, et al. Some Socioeconomic Factors and Lifestyle Habits Influencing the Prevalence of Obesity among Adolescent Male Students in the Hail Region of Saudi Arabia. Children, 2018; 5(3): 1-10.

3. BASTO F, et al. Relação entre atividade física e desportiva, níveis de IMC, perceções de sucesso e rendimento escolar. Motricidade, 2015; 11(3): 41-58.

4. BARBOSA SC, et al. Ambiente escolar, comportamento sedentário e atividade física em pré-escolares. Revista Paulista de Pediatria, 2016; 34(3): 301-308.

5. BLOCH KV, et al. ERICA: prevalência de hipertensão arterial e obesidade em adolescentes brasileiros. Revista de Saúde Pública, 2016; 50(1): 1-13.

6. BRASIL. Ministério da Saúde. Orientações para a coleta e análise de dados antropométricos em serviços de saúde: Norma Técnica do Sistema de Vigilância Alimentar e Nutricional - SISVAN. 1ed. rev. e atual. Brasília: Ministério da Saúde, 2011, 76p. Disponível em: < http://bvsms.saude.gov.br/bvs/publicacoes/orientacoes_coleta_analise_dados_antropometricos.pdf>. Acesso em: 28 abr. 2019.

7. CUREAU FV, et al. ERICA: inatividade física no lazer em adolescentes brasileiros. Revista de Saúde Pública, 2016; 50(1): 1-11.

8. D' AVILA GL, et al. associação entre consumo alimentar, atividade física, fatores socioeconômicos e percentual de gordura corporal em escolares. Ciência e Saúde Coletiva, 2016; 21(4): 1171-1081.

9. DUMITH SC, et al. Prática de atividade física entre jovens em município do semiárido no Brasil. Ciência e Saúde Coletiva, 2016; 21(4): 1083-1093.

10. GURNANI M, et al. Childhood Obesity: Causes, Consequences, and Management. Pediatric Clinics of North America, 2015; 10(3): 44-234.

11. INSTITUTO BRASILEIRO DE GEOGRAFIA E ESTATÍSTICA - IBGE. Pesquisa de orçamentos familiares 20082009: antropometria e estado nutricional de crianças, adolescentes e adultos no Brasil. ver. e atual. Rio de Janeiro: IBGE,2010, 130p. Disponível em: < https://biblioteca.ibge.gov.br/visualizacao/livros/liv45419.pdf>. Acesso em: 19 abr. 2019.

12. KNEIPP C, et al. Excesso de peso e variáveis associadas em escolares de Itajaí, Santa Catarina, Brasil. Revista de Saúde Pública, 2015; 20(8) 2411-2422.

13. KUMAR S, KELLY AS. Review of Childhood Obesity. Mayo Clinic Proceedings, 2017; 92(2): 251-265.

14. RECH RR, HALPERN R. Obesidade infantil: perfil epidemiológico e fatores associados. Rio Grande do Sul: Educs, 2011. 
15. SANTOS MM, et al. Dietary Knowledge, Behaviours and Attitudes of Students of Public School in a Northeastern City in Brazil. Scientific Research, 2014; 6(11): 1315-1321.

16. SAHOO K, et al. Childhood obesity: causes and consequences. Journal of Family Medicine and Primary Care, 2015; 4(2): 87-192.

17. SOUSA S, et al. Deslocamento Ativo para a escola. Percepções positivas e negativas de crianças de uma escola u1rbana de Vila Nova de Famalicão - Portugal. In: VI Congresso Internacional de Pedagogia do Esporte, 5., 2015, Maringá. Anais [...]. Maringá: Universidade Estadual de Maringá (UEM), 2015, p. 1-7.

18. MONTEIRO AR, et al. excesso de peso entre jovens de um município do semiárido brasileiro: estudo de base populacional. Ciência e Saúde Coletiva, 2016; 21(4): 1157-1164.

19. MUSAIGER AO, KALAN F. Dietary habits and lifestyle among adolescents in Damascus, Syria. Annals of Agricultural and Environmental Medicine, 2014; 21(2): 416-419.

20. 2NETO ACB, et al. Peso corporal e escores de consumo alimentar em adolescentes no nordeste brasileiro. Revista Paulista de Pediatria, 2015 33(3): 318-325

21. OLIVEIRA JS, et al. ERICA: uso de telas e consumo de refeições e petisco por adolescentes brasileiros. Revista de Saúde Pública, 2016; 50(1): 1-9.

22. PANAZZOLO PR, et al. Prevalência de sobrepeso e obesidade em escolares do município de Feliz, Rio Grande do Sul, Brasil. Revista Brasileira de Medicina de Família e Comunidade, 2014; 9(31): 142-148.

23. PINTOS RP, et al. Análise de fatores associados ao excesso de peso em escolares. Revista Paulista de Pediatria, 2016; 34(4): 460-468.

24. RANGEL SRV, et al. Atividade física e comportamento sedentário: prevalência e fatores associados em adolescentes de três escolas públicas de pelotas/RS. Revista Biomotriz, 2015; 9(1): 1-17.

25. RAMOS MLM, et al. Sobrepeso e obesidade em escolares de 10 a 14 anos. Revista Brasileira em Promocão da Saúde, 2013; 26(2); 223-232.

26. REINALDO, EDF, et al. Mudanças de hábitos alimentares em comunidades rurais do semiárido da região nordeste do Brasil. Asociación Interciencia, 2015; 40(5): 330-336.

27. SOUZA AM, et al. ERICA: ingestão de macro e micronutrientes em adolescentes brasileiros. Revista de Saúde Pública, 2016; 50(1): 1-15.

28. SOUZA S, et al. Modos de deslocamento para a escola em crianças de escolas do meio rural e urbano. Câmara Municipial de Idanha-a-nova: Castelo Branco, 2018.

29. THE NS, et al. Association of adolescent obesity with risk of severe obesity inadulthood. JAMA, North Carolina, 2010; 304(18): 2042-2047.

30. WANG S, et al. Trends in overweight and obesity among Chinese children of 7-18 years old during 1985-2014. Chinese journal of preventive medicine, 2017; 51(4): 300-305.

31. WORLD HEALTH ORGANIZATION - WHO. Obesity: Preventing and managingthe global epidemic. Report of a WHO consultation on Obesity. Geneva: Avenue Appia, 2000.

32. WORLD HEALTH ORGANIZATION - WHO. Obesity and overweight. 2018. Disponível em:<https://www.who.int/en/news-room/fact-sheets/detail/obesity-and-overweight>. Acesso em: 22 jan. 2019. 